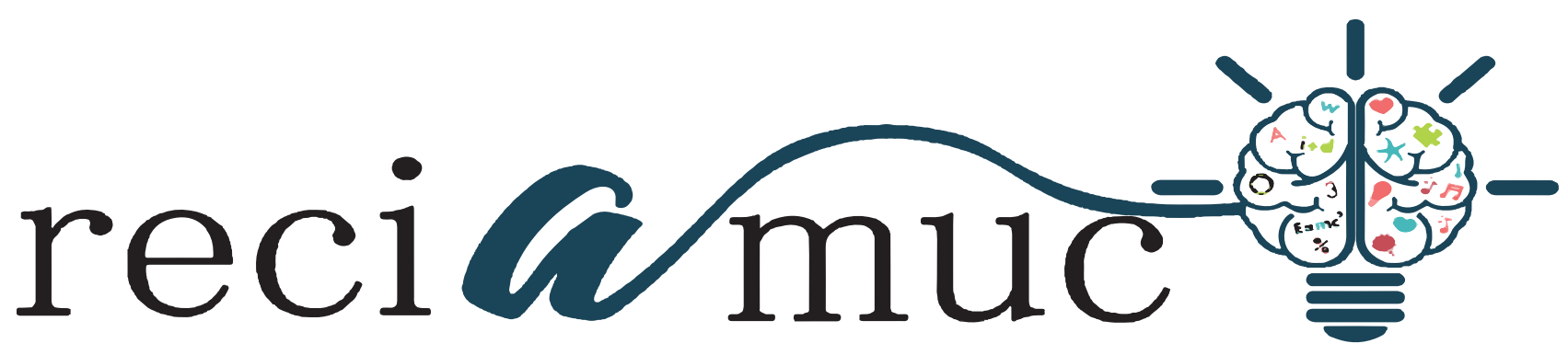

DOl: 10.26820/reciamuc/4.(3).julio.2020.80-89

URL: https://reciamuc.com/index.php/RECIAMUC/article/view/501

EDITORIAL: Saberes del Conocimiento

REVISTA: RECIAMUC

ISSN: 2588-0748

TIPO DE INVESTIGACIÓN: Artículo de Revisión

Código UNESCO: 32 Ciencias Médicas; 3201 Ciencias Clínicas

PAGINAS: $80-89$

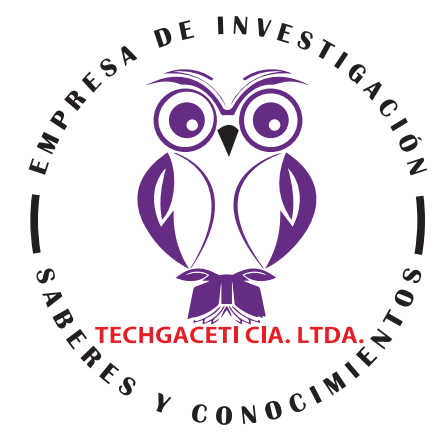

\title{
Utilidad del antígeno prostático específico cáncer de próstata
}

Usefulness of prostate specific antigen for prostate cancer

Utilidade do antígeno específico da próstata para o câncer de próstata

Patricia Herminia Corella Sanguil'; Javier Mauricio Martínez Otálora2;

Yessica Viviana Hernández Acosta ${ }^{3}$ D Darlys Tatiana Cerón Pérez

RECIBIDO: 20/05/2020 ACEPTADO: 19/07/2020 PUBLICADO: 30/07/2020

1. Médico Cirujano; Investigador Independiente; Quito, Ecuador; patylu567@hotmail.com; (iD https://orcid.org/0000-00031240-6676

2. Médico General; Investigador Independiente; Bogotá, Colombia; javiermarrothmd@gmail.com; (iD https://orcid.org/00000002-2445-5259

3. Médico General; Investigador Independiente; Bogotá, Colombia; yesshernandez777@gmail.com; iD https://orcid.org/0000-0002-2108-961X

4. Médico General; Investigador Independiente; Popayán-Cauca, Colombia; taticeron18@gmail.com; (iD https://orcid. org/0000-0001-8454-0968

CORRESPONDENCIA

Patricia Herminia Corella Sanguil

patylu567@hotmail.com

Quito, Ecuador

○ RECIAMUC; Editorial Saberes del Conocimiento, 2020 


\title{
RESUMEN
}

La utilidad del antígeno prostático específico (APE, o PSA, por su abreviatura en inglés) para cáncer de próstata se destacado desde hace más de 30 años, por tratarse de un biomarcador característico tanto en el diagnóstico de este tipo de tumores como en el monitoreo de la reacción del mismo ante diversas terapias. Con la presente revisión se busca rescatar aquellos recursos con los cuales se pueda exponer sobre la utilidad del APE (PSA) para el cáncer de próstata y ciertos aspectos controvertidos en torno al tema. Es por ello que, en base al criterio de expertos, en los resultados se reseñan algunas las acepciones vigentes, las ventajas y desventajas de la prueba del APE y la utilidad que sobre la misma es posible extraer del desarrollo y las conclusiones de investigaciones particulares. Se concluye indicando que la utilidad del APE (PSA) para el cáncer de próstata se ha discutido ampliamente desde hace ya más de 20 años, y hasta ahora es posible atreverse a asegurar por una parte que, si bien es válido que, este tipo de medición sea clínicamente recomendada a nivel individual por el peso de sus beneficios, no debe relegarse la considerable brecha de imprecisión que ésta prueba, de manera "aislada" en una sola analítica, representa si se considerara en un cribado, lo que por cierto se vincula con un posible sobrediagnóstico, es decir, que se "detecte" un cáncer cuando no en realidad lo hay, o por el contrario, se infradiagnóstique. En todo caso, la recomendación generalizada es valerse de las múltiples herramientas y pruebas complementarias de las que se disponen actualmente, las cuales incrementan considerablemente la especificidad del diagnóstico oportuno.

Palabras clave: Biomarcador, especificidad, cribado, sobrediagnóstico, infradiagnóstico.

\begin{abstract}
The usefulness of prostate specific antigen (PSA, or PSA, for its abbreviation in English) for prostate cancer has been highlighted for more than 30 years, as it is a characteristic biomarker both in the diagnosis of this type of tumors and in monitoring of its reaction to various therapies. This review seeks to rescue those resources with which it is possible to present the usefulness of PSA (PSA) for prostate cancer and certain controversial aspects of the subject. That is why, based on the criteria of experts, the results outline some of the current meanings, the advantages and disadvantages of the APE test and the usefulness that can be derived from the development and conclusions of particular investigations. It concludes by indicating that the utility of PSA (PSA) for prostate cancer has been widely discussed for more than 20 years, and until now it is possible to dare to assure on the one hand that, although it is valid that this type of measurement is clinically recommended at the individual level due to the weight of its benefits, the considerable imprecision gap that this test, in an "isolated" way in a single analytic, does not have to be relegated if it were considered in a screening, which is certainly linked with a possible overdiagnosis, that is, that a cancer is "detected" when there is not really one, or on the contrary, it is underdiagnosed. In any case, the general recommendation is to use the multiple tools and complementary tests currently available, which considerably increase the specificity of the timely diagnosis.
\end{abstract}

Keywords: Biomarker, specificity, screening, overdiagnosis, underdiagnosis.

\section{RESUMO}

A utilidade do antígeno prostático específico (PSA, ou PSA, por sua abreviatura em inglês) para o câncer de próstata tem sido destacada há mais de 30 anos, por ser um biomarcador característico tanto no diagnóstico desse tipo de tumor quanto no monitoramento de sua reação a várias terapias. Esta revisão busca resgatar aqueles recursos com os quais é possível apresentar a utilidade do PSA (PSA) para o câncer de próstata e alguns aspectos controversos do assunto. É por isso que, com base em critérios de especialistas, os resultados delineiam alguns dos significados atuais, as vantagens e desvantagens do teste APE e a utilidade que pode ser derivada do desenvolvimento e conclusões de investigações particulares. Conclui indicando que a utilidade do PSA (PSA) para o câncer de próstata vem sendo amplamente discutida há mais de 20 anos, e até agora é possível ousar afirmar que, embora seja válido que este tipo de medida é clinicamente recomendado a nível individual devido ao peso de seus benefícios, a considerável lacuna de imprecisão de que esse teste, de forma "isolada" em uma única analítica, não deva ser relegado se fosse considerado em uma triagem, que é certamente ligada a um possível sobrediagnóstico, ou seja, que um câncer seja "detectado" quando realmente não existe, ou ao contrário, é subdiagnosticado. Em todo caso, a recomendação geral é a utilização das múltiplas ferramentas e exames complementares disponíveis atualmente, que aumentam consideravelmente a especificidade do diagnóstico oportuno.

Palavras-chave: Biomarcador, especificidade, rastreamento, sobrediagnóstico, subdiagnóstico. 


\section{Introducción}

Dellavedova (2016) al comenzar su estudio fundadamente refirió que, la utilidad del antígeno prostático específico (APE, o PSA, por su abreviatura en inglés) para cáncer de próstata se destacado desde hace más de 30 años, por tratarse de un biomarcador característico tanto en el diagnóstico de este tipo de tumores como en el monitoreo de la reacción del mismo ante diversas terapias. "También es de destacar que tiene valor predictivo para cáncer de próstata, y esta predicción sirve tanto para medir el riesgo actual de desarrollar el tumor como en el futuro." (pág. 20)

Asociación Española Contra el Cáncer (AECC) asegura que APE es una proteína producida en la próstata casi exclusivamente, y que es segregada en altas concentraciones junto con el semen con el objeto de diluir el semen eyaculado y a la vez acondicionando el medio idóneo para un libre desplazamiento de los espermatozoides. Acotan que sólo una mínima cantidad de ésta proteína es la que logra filtrarse en el torrente sanguíneo que es el que en definitiva puede ser determinado por medio de un test de laboratorio elemental. Así mismo afirman que esta prueba se ha considerado como uno de los métodos diagnósticos que más favorece en el establecimiento de una sospecha de cáncer de próstata al consistir, sí misma, el más alto valor predictivo de esta patología, sin embargo, no dejan de un lado resaltar que "el PSA es específico de la glándula prostática, pero no de cáncer de próstata y puede encontrarse elevado por otras causas" tales como: prostatitis, infecciones y otras comorbilidades. (AECC, 2018)

Por su parte, Duraj (2019) menciona otro importante aspecto entorno a dicho antígeno prostático ya que, al recordar que fue hace casi 35 años que la Administración Estadounidense de Alimentos y Fármacos (FDA, por sus siglas en inglés) aprobó el uso del APE (PSA) como el primer biomarcador tumoral, con el cual se debía perseguir solamente evaluar el progreso y la respuesta al tratamiento del cáncer de próstata; destacando éste autor que para la fecha aún no se hacía referencia a la detección de cáncer o "cribado" mediante APE. Éste enfoque precisamente surgió; junto con el tacto (examen) rectal digital (ERD), a partir de 1994, no obstante, ésta práctica recientemente ha sido el centro de serias controversias, en razón de su eficacia y la repercusión real en la práctica clínica.

El análisis de sangre de APE aún se concibe como una de las dos primeras pruebas (la otra se trata del ERD) con la cual se puede detectar el cáncer de próstata. (Vachani, 2020)

Esta experta no difiere en cuanto a la acepción que, frecuentemente, coinciden otros tratadistas respecto a lo que significa la prueba de APE para el cáncer de próstata, sin embargo, puntualiza que dicho examen no es infalible ya que se dan los casos en los que ciertos tumores no elevan el nivel de APE en la sangre, pero otros procesos; como la hiperplasia prostática benigna y la prostatitis, si pueden generar un incremento de dicha proteína falsamente. Insistió que, el término "específico" al que se hace referencia cuando se habla de "antígeno específico de la próstata" debe entenderse como un indicador específico para la glándula prostática más no para el cáncer de próstata; de todas maneras, en términos de probabilidad, la causa de un elevado nivel de PSA se asocia con el cáncer de próstata.

Hay una gran cantidad de pruebas especializadas de PSA que se utilizan para ayudar a diferenciar entre PSA elevado debido a condiciones benignas y aquellas debidas al cáncer de próstata, o para determinar la probabilidad de cáncer y la necesidad de una biopsia.

- La prueba de PSA libre evalúa la relación entre el PSA que está libre en sangre y el PSA que está unido a las proteínas en sangre. En casi todos los hombres, la 
mayoría del PSA está ligado a proteínas en sangre y el porcentaje de PSA libre generalmente es más bajo en hombres con cáncer de próstata.

- Un examen llamado PSA complejo mide la forma compuesta del PSA. La ventaja es que mientras que la prueba de PSA libre requiere dos pruebas en el laboratorio, la prueba compleja requiere sólo una.

- La prueba tradicional de PSA es una combinación de PSA libre y PSA complejo.

- La velocidad del PSA se utiliza para describir la velocidad a la que aumenta el valor del PSA en una serie de análisis de sangre (y en el tiempo). El resultado se reporta como una cantidad $(\mathrm{ng} / \mathrm{ml})$ por año. Se necesita una serie de tres pruebas durante un período mínimo de 18 meses para calcular la velocidad del PSA.

- El tiempo de duplicación del PSA es el período de tiempo que tarda el PSA en duplicarse.

- La densidad del PSA se utiliza para evaluar el nivel de PSA en relación con el tamaño total de la glándula prostática. Se realiza una ecografía transrectal para determinar el tamaño de la próstata y ese número se divide en el valor del PSA. La teoría es que un aumento en el PSA puede ser directamente proporcional a un aumento en el tamaño de la próstata. Esta prueba no se utiliza muy a menudo, ya que no se ha demostrado que sea fiable, mientras que otras pruebas son más fiables y menos costosas. Una de las mayores preocupaciones es la poca fiabilidad del ultrasonido transrectal para medir correctamente el volumen de la próstata. (Vachani, 2020)

Con el presente estudio se pretende revisar parte importante de la literatura cientificoacadémica reciente que se encuentra dis- ponible completa y libremente, a fines de rescatar aquellos recursos con los cuales se pueda exponer sobre la utilidad del APE (PSA) para el cáncer de próstata y ciertos aspectos controvertidos en torno al tema. A continuación, se explica la metodología de investigación aplicada, y se prosigue con las reseñas tras los resultados obtenidos, alusivos en sentido amplio a las acepciones vigentes, la utilidad que posee la prueba del APE para el cáncer de próstata según el criterio de varios expertos tras el desarrollo de sus investigaciones particulares y las conclusiones que de éstos fue posible extraer.

\section{Materiales y Métodos}

La presente investigación ha sido adaptada a una de diseño documental y a su vez enmarcada en una metodología de revisión, ya que, como inicialmente se ha referido, el objeto perseguido no es otro que el de reseñar las propias ideas que algunos entendidos ofrecen mediante el desarrollo de literatura cientificoacadémica entorno a la utilidad del APE en el cáncer de próstata.

La búsqueda fue iniciada en la penúltima semanada de Julio 2020, fundamentalmente mediante el uso de bases de datos tales como: Medigraphic, IntraMed, Elsevier, PubMed, BVS (LILACS), IBECS y Medes; así como también en otros portales web de organizaciones, asociaciones, instituciones, fundaciones y sociedades de carácter internacional, regional e incluso nacional, enfocadas en el área de la salud, medicina humana y oncología.

Cada una de las pesquisas se hicieron individualmente en cada sitio web, utilizando como principal criterio de búsqueda las siguientes sintaxis "utilidad and APE or PSA and cáncer próstata" y "utility or usefulness and PSA and prostate cancer" con las que se obtuvo una variedad de resultados alusivos a diversos tipos de fuentes bibliográficas. 
Los enlaces obtenidos oscilaron entre 1 y más de 700000, por lo que, en aras de alcanzar resultados simplificados, necesariamente se tuvieron que refinar aplicando otros criterios de selección, entre los cuales figuraron: idioma español e inglés, periodo de publicación en los últimos 10 años, acceso libre y completo, mayor correlación o especificidad temática, relevancia investigativa, tipo de recurso bibliográfico, priorizando entre éstos: artículos científicos originales y de revisión (con y sin metadatos) protocolos, consensos, manuales de procedimiento, guías clínicas, boletines informativos, ensayos, tesis de grado, posgrado o doctorado, informes y proyectos, directrices médicas, folletos, resúmenes, libros digitalizados (o electrónicos) y demás tipos de recursos bibliográficos en formato digital; siendo paralelamente descartados los recursos repetidos y otros que, básicamente, no cumplieran con las características antes descritas.

Luego del proceso antes descrito, entre los mismos integrantes del equipo, se efectuó la lectura, análisis e interpretación de todo el material documentado que en definitiva fue seleccionado, debiendo entenderse esta fase como, el desarrollo de lecturas individuales y grupales que derivaron en discusiones y finalmente en consensos que sirvieron para compilar y plasmar el contenido expuesto en la presente entrega.

\section{Resultados}

De conformidad al Instituto Nacional del Cáncer de los Estados Unidos de América ( $\mathrm{NCl}$, por su abreviatura en inglés):

El antígeno prostático específico, o PSA, es una proteína producida por las células normales y malignas de la glándula prostática. El análisis del PSA mide la concentración del PSA en la sangre de un hombre. Para esa prueba, se envía una muestra de sangre a un laboratorio para ser analizada. Los resultados se reportan generalmente en nanogramos de PSA por cada mililitro de sangre $(\mathrm{ng} / \mathrm{ml})$. ( $\mathrm{NCl}, 2017)$
Al APE se le conoce también como: calicreína III, seminina, semenogelasa, $\gamma$-seminoprotein o antígeno P30, y consiste en una glucoproteína especialmente sintetizada por las células epiteliales de la próstata con la finalidad de que esta actúe como disoluble del coágulo seminal. Ha de destacarse que la reducción o incremento del valor de ésta en sangre (en la que por cierto se puede hallar en magnitudes proporcionalmente minúsculas en comparación a como se encuentra en el líquido semial) pueden encontrarse vinculados a distintas y regulares fases biológicas que se den a nivel prostático, y que por lo general son benignas, de allí es que se deriva la naturaleza esta proteína próstata-específica, por lo que no debe asumirse como una cáncer-específica. (Duraj, 2019)

En la Guía de Práctica Clínica para la detección temprana, diagnóstico, tratamiento, seguimiento y rehabilitación del cáncer de próstata (Colciencias \& Ministerio de Salud y Protección Social de Colombia, 2013) se deja claro que el APE es una proteína generada por la misma próstata, y que su medición a ciertos niveles no se considera un indicador exclusivo para el diagnóstico de cáncer de próstata, es decir, sus niveles pudieran estar alterados a raíz de otras enfermedades de la próstata. El análisis de sangre que se llevan a cabo en un laboratorio por medio del cual se logra medir los niveles de dicha proteína se lleva a cabo tomando la muestra en ausencia de infecciones en curso (prostatitis), biopsias previas de la próstata e instrumentación reciente y eyaculaciones recientes, ya son situaciones que directamente alteran los niveles del antígeno prostático. Adicionalmente se destaca la importancia de que la evaluación de estos niveles sea hecha por un especialista y de manera individual en cada paciente, atendiendo su edad y características propias, puesto que tales condiciones pueden estar asociadas a la anormalidad en los resultados de medición de estos niveles, de allí la necesidad de repetir esta prueba en 
ciertos casos.

No obstante, entorno a APE, fundadamente se hace referencia a sus altos rangos de especificidad y sensibilidad (entre 72,1 y $93,2 \%$, ) que, al ser cercanamente comparables con los obtenidos mediante el tacto rectal, son los que se sugieren se usen de manera conjunta en escenarios clínicos en lugar de poblacionales. De la misma manera en la obra se señala un especial interés en torno a considerables tasas $(75,9 \%)$ de falsos positivos del PSA (al igual que el examen digital rectal $82,5 \%$ ) y de sobretratamiento, con los consiguientes efectos urológicas. (pág. 67)

Según Vachani (2020) lo que se considera "normal" respecto al nivel de APE en sangre es un valor inferior a $4.0 \mathrm{ng} / \mathrm{ml}$, pero, enfatiza que la tendencia del PSA a lo largo del tiempo es lo que realmente importa mucho antes que un solo valor de PSA.

Los estudios han demostrado que los hombres que tienen un aumento constante en su PSA son más propensos a tener cáncer de próstata y, si el aumento es rápido, es más probable que sea un cáncer agresivo.

Adicionalmente, la especialista refiere que, de acuerdo con Sociedad Norteamericana contra el Cáncer (ACS, por su abreviatura en inglés) se logró un consenso en cuanto a que, existirá un mayor riesgo de cáncer de próstata (superior al $50 \%$ de probabilidad) en aquellos hombres que tengan un nivel total de PSA por encima de $10.0 \mathrm{ng} / \mathrm{ml}$.

Hasta aproximadamente 2008, algunos médicos y organizaciones profesionales alentaron la detección anual de PSA para hombres a partir de los 50 años. Algunas organizaciones recomendaron que los hombres con mayor riesgo de cáncer de próstata, incluidos los hombres y hombres afroamericanos cuyo padre o hermano tenían cáncer de próstata, comenzaran la detección. a la edad de 40 o 45 años. Sin embargo, a medida que se aprendió más sobre los beneficios y los daños del examen de detección de cáncer de próstata, varias organizaciones comenzaron a advertir contra el examen de rutina de la población. La mayoría de las organizaciones recomiendan que los hombres que están considerando la detección de PSA primero discutan los riesgos y beneficios con sus médicos.

[...] No hay un nivel específico normal o anormal de PSA en la sangre, y los niveles pueden variar con el tiempo en el mismo hombre. En el pasado, la mayoría de los médicos consideraban que los niveles de PSA de 4.0 ng / mL y más bajos eran normales. Por lo tanto, si un hombre tenía un nivel de PSA superior a 4.0 ng / mL, los médicos a menudo recomendarían una biopsia de próstata para determinar si el cáncer de próstata estaba presente.

Sin embargo, estudios más recientes han demostrado que algunos hombres con niveles de PSA inferiores a $4.0 \mathrm{ng} / \mathrm{ml}$ tienen cáncer de próstata y que muchos hombres con niveles más altos no tienen cáncer de próstata. Además, varios factores pueden hacer que el nivel de PSA de un hombre fluctúe. Por ejemplo, el nivel de PSA de un hombre a menudo aumenta si tiene prostatitis o una infección del tracto urinario. Las biopsias de próstata y la cirugía de próstata también aumentan el nivel de PSA. Por el contrario, algunos medicamentos, como la finasterida y la dutasterida, que se usan para tratar la HPB, disminuyen el nivel de PSA de un hombre. El nivel de PSA también puede variar algo entre los laboratorios de prueba. (NCl, 2017)

\section{Ventajas y desventajas de la prueba del APE (PSA)}

Ventajas

- La prueba del APE podría favorecer la detección temprana del cáncer de próstata.

- Facilita el tratamiento del cáncer e incrementa las probabilidades de curación (a nivel individual) si se diagnostica en los 


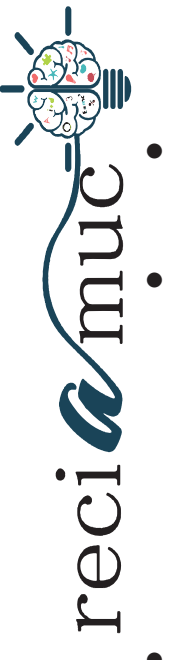

estados tempranos de la enfermedad y se localiza.

- Se puede realizar con un simple análisis de sangre ampliamente disponible.

En los hombres que valoran el saber sobre el no saber sobre el diagnóstico de esta enfermedad, puede brindar cierta tranquilidad, ya que, si se le diagnostica, puede tratarlo a tiempo, y si no se le diagnostica, gozara con plena confianza de su buen estado de salud.

- Desde que esta prueba está disponible, la mortalidad por cáncer de próstata ha sido disminuida.

- En caso de duda diagnóstica, es posible vigilar los niveles de PSA y valorar su evolución a lo largo del tiempo.

(Clínica Mayo, 2019; Duraj, 2019)

Desventajas

- Algunos cánceres de próstata crecen lentamente y nunca se extienden más allá de la glándula prostática.

- No todos los cánceres de próstata necesitan tratamiento. Es posible que el tratamiento para el cáncer de próstata tenga riesgos y efectos secundarios, incluyendo incontinencia urinaria, disfunción eréctil o disfunción intestinal

- Las pruebas del APE no son infalibles. Es posible que los niveles de APE estén elevados sin haber un cáncer presente, y que no estén elevados cuando sí lo hay. (falsos positivos y falsos negativos)

- Un diagnóstico de cáncer de próstata puede provocar ansiedad y confusión.

- La prueba del APE ha reducido las muertes, pero el número podría no ser considerablemente suficiente para justificar el costo y la posibilidad de daño a la persona que se somete a la prueba.

(Clínica Mayo, 2019; Duraj, 2019)

\section{Utilidad del APE para el cáncer de prós- tata}

En 2007, Uribe argumentaba que, a pesar de la reconocida utilidad que en general concedía el uso del APE como marcador de cáncer más solicitado a nivel mundial por los médicos,

su interpretación está sometida a una aguda controversia, no muy justificada puesto que a menudo los conflictos se generan por desconocer la naturaleza del antígeno como subproducto de la glándula prostática y de su dinámica como marcador de la salud del órgano. (Uribe, 2007, pág. 37)

En el estudio de Ormachea, Sánchez, Callisaya, \& Salcedo (2011), el objetivo consistió en hallar la relación de los niveles de APE total con los diagnósticos de cáncer de próstata e hipertrofia benigna (como diagnósticos presuntivos) mediante la comparación de muestras procesadas por el método de fluorimetría en el Laboratorio central del Hospital Obrero. Respecto al cáncer de próstata, de esta investigación fue posible extraer que:

[...] En lo referente al cáncer prostático mostró 84 (19.90\%) diagnósticos, de los cuales $29(34,5 \%)$ casos presentaron niveles de PSA por encima de lo normal, de estos 15 presentaron niveles de PSA altamente elevados, 4 moderadamente elevados y 10 ligeramente elevados, mostrando una relación con datos bibliográficos que indican un $26.9 \%$ de casos con PSA elevado revelan la presencia de cáncer prostático. (pág. 43)

De lo anterior puede inferirse que, en base a los objetivos planteados, en el estudio es posible evidenciar efectivamente la relación que se da entre concentraciones elevadas de APE con los diagnósticos de cáncer prostático ya que, la proporción de la población estudio que resultó con índices de APE superiores a los normales coincidieron con lo indicado en las referencias bibliográficas en las que se apoyó teóricamente dicho 
estudio, de allí pues podría decir que este bien podría servir como un primer ejemplo en el que claramente queda demostrada la utilidad del APE en el cáncer de próstata.

En un estudio retrospectivo (Viveros et al., 2015) de los expedientes de 137 pacientes a quienes se practicó prostatectomía radical retropúbica, entre Abril de 1991 y Julio de 2005, por adenocarcinoma de próstata (confirmado histológicamente por biopsia transrectal) clínicamente órgano confinado, se proyectó evaluar la utilidad del porcentaje de APE-FL para pronosticar carcinoma de próstata localmente avanzado o invasión perineural en pacientes sometidos a prostatectomía radical retropúbica por carcinoma de próstata clínicamente organoconfinado ya que según sus los investigadores, fue demostrado a finales de 1990 "que el uso del porcentaje de la fracción libre del APE (APE-FL) es útil cuando el APE total (APE-T) se encuentra entre 4 y 10", y ello fue aunado al hecho de que, cerca de esa misma época, se había evidenciado que dicho porcentaje era de igual utilidad diagnóstica cuando el APE-T se encuentra entre 2.5 y 4.5 .

En los resultados de éste obra, se dejó evidencia de que fue posible localizar la enfermedad en el $59 \%$ de los casos, se detectó extensión extracapsular en un 32\%, la invasión a vesículas seminales se dio en el 9\% de ellos y hacia los ganglios en el 3\% de los estudiados. Los valores promedio de APE-FL en casos de enfermedad confinada al órgano fue $16 \%$ y $12 \%$ en tumores localmente avanzados; con APE-FL 15\% en $50 \%$ y $86 \%$, respectivamente. El riesgo de estadio localmente avanzado con APEFL 15\% fue 3 veces mayor, en comparación con la APE-FL >15\%. Sin embargo,

Al analizar la asociación entre APE-FL menor o igual a 15\% y la invasión perineural postoperatoria, se encontró una asociación 2 veces mayor (intervalo de confianza 0.3711.02) en los pacientes con APE-FL menor o igual al 15\%, sin significación estadística $(p=0.35)$.
En definitiva, tras ese estudio lograron observar una relación ajustada entre el porcentaje de APE-FL y signos patológicos adversos.

Contrario a los estudios antes reseñados, en la literatura revisada también se ha encontrado un trabajo investigativo de España (Panach, Gironés, Sánchez, Doménech, \& Martínez, 2017) en el que se deja en evidencia la poca utilidad del APE para el cáncer de próstata. Allí se fundamenta sobre la existencia de investigaciones a largo plazo en las que se pone en tela de juicio la utilidad del APE específicamente como herramienta de cribado, tales casos son representados por los ya conocidos dos grandes estudios prospectivos en Estados Unidos de América y Europa, idea que, por cierto, también se puede deducir en (AECC, 2018).

Lo que ha interesado destacar entre los objetivos propuestos en el estudio de Panach et al. (2017) a sido el aspecto relacionado con "las creencias subjetivas sobre su utilidad". En ese sentido, mediantes sus resultados los autores señalaron que:

Sobre la creencia subjetiva de los médicos de primaria en torno al PSA, se debe destacar que un $64,1 \%$ se ha planteado muchas veces la verdadera utilidad del PSA, y un $29,1 \%$ cree que es poco útil para diagnosticar cáncer.

[...] En la línea de esta creencia sobre la dudosa utilidad del PSA van otros datos, como el hecho de que un $27,5 \%$ de médicos varones afirme que no se realizaría una determinación de PSA para el diagnóstico precoz de cáncer de próstata, y un 22,5\% de pendiendo de la situación. Este hecho podría relacionarse con las conclusiones de otros estudios, donde se afirma que el principal motivo por el que los médicos de atención primaria solicitan el PSA es por demanda o preocupación del paciente, independientemente de la indicación clínica o un convencimiento firme sobre su utilidad. (págs. 191, 193-194) 
La Fundación Femeba (2020) refiere (Gilbert \& Albertsen, 2020) que:

La mayoría de los cánceres de próstata detectados por PSA actúan más como una enfermedad crónica que como una neoplasia maligna agresiva. La mayoría son cánceres bien diferenciados, es decir, en un grupo con bajo puntaje de Gleason. Algunos están en el otro extremo del espectro: cánceres pobremente diferenciados en un grupo de puntaje alto de Gleason. Los hombres con enfermedad poco diferenciada a menudo mueren dentro de los 10 años posteriores al diagnóstico, a menudo a pesar de someterse a radiación, cirugía o ambos. La detección de PSA encuentra principalmente cánceres de próstata bien diferenciados; los cánceres poco diferenciados, los que generalmente matan a los hombres, se encuentran con mucha menos frecuencia. De hecho, algunos hombres con los cánceres de próstata menos diferenciados y mortales tienen un nivel normal de PSA.

Esta organización definitivamente no se encuentra a favor de la práctica contemporánea de detección con PSA, especialmente al considerar el sistema de atención médica en el que participa, que se basa en el volumen, a su vez reconociendo que tal postura representa un juicio de valor. No obstante, subrayan que se dan los casos de personas que reciben un beneficio sustancial (al evitar la muerte por cáncer de próstata), pero también existen aquellos en los que muchas más están expuestas a biopsias innecesarias, operaciones y otra fuente de estrés financiero. Reconocen además que tales beneficios y daños, por su propia naturaleza y por el momento, no es posible medirlos bajo una misma medida; es por ello que, generar una respuesta "correcta" de manera unánime, es igualmente inviable por falta de un modelo de cálculo.

\section{Conclusión}

Mediante la presente revisión es posible deducir que, sobre la utilidad del APE (PSA) para el cáncer de próstata se ha discutido bastamente desde hace ya más de 20 años, y hasta ahora es posible atreverse a asegurar por una parte que, si bien es válido que, este tipo de medición sea clínicamente recomendada a nivel individual por el peso de sus beneficios, no debe relegarse la considerable brecha de imprecisión que ésta prueba, de manera "aislada" en una sola analítica, representa si se considerara en un cribado, lo que por cierto se vincula con un posible sobrediagnóstico, es decir, que se "detecte" un cáncer cuando no en realidad lo hay, o por el contrario, se infradiagnóstique. En todo caso, la recomendación generalizada es valerse de las múltiples herramientas y pruebas complementarias de las que se disponen actualmente, las cuales incrementan considerablemente la especificidad del diagnóstico oportuno.

Así mismo se ha encontrado referencias en torno al desarrollo de marcadores nuevos, o índices de marcadores más sensibles y específicos que coadyuvan a los profesionales de la salud en la toma decisiones con una certeza diagnóstica mayor. Respecto a éstos cabe recordar que, independientemente de la precisión asociada, aún no se recaban resultados validos en grandes cohortes de pacientes.

Paralelamente ha de considerarse que recientemente, mediante un metaanálisis, se obtuvo un hallazgo muy importante que se ha considerado bien vale la pena mencionar, y es que, se evidenció que la terapia hormonal adyuvante se asocia con una reducción del 30\% en la mortalidad por cáncer de próstata.

\section{Bibliografía}

AECC. (2018). Cáncer de próstata. Recuperado el 25 de 07 de 2020, de https://www.aecc.es/es/todo-sobre-cancer/tipos-cancer/cancer-prostata/ PSA

Clínica Mayo. (20 de Junio de 2019). ¿Examen de detección de cáncer de próstata: ¿Deberías someterte a una prueba de APE? Recuperado el 25 de 07 de 2020, de https://www.mayoclinic.org/eses/diseases-conditions/prostate-cancer/in-depth/ prostate-cancer/art-20048087?p=1 
Colciencias \& Ministerio de Salud y Protección Social de Colombia. (Junio de 2013). Guía de práctica clínica (GPC) para la detección temprana, diagnóstico, tratamiento, seguimiento y rehabilitación del cáncer de próstata. Recuperado el 25 de 07 de 2020, de http://gpc.minsalud.gov.co/gpc_sites/Repositorio/Conv_500/GPC_cancer_prostata/ gpc_cancer_prostata_completa.aspx

Dellavedova, T. (Enero-Febrero de 2016). Antígeno prostático específico. Desde sus inicios hasta su reconocimiento como biomarcador de cáncer de próstata. Archivos Españoles de Urología, 69(1), 19-23. Recuperado el 25 de 07 de 2020, de https://aeurologia.com/article_detail.php?aid=aeebb6b545a4cb8236ec182a22fb353dde1e9117

Duraj, T. (18 de Diciembre de 2019). PSA en detalle: ¡todo lo que necesitas saber para prevenir el cáncer de próstata! Recuperado el 25 de 07 de 2020, de https://www.melio.es/issue/psa-en-detalle-todo-lo-que-necesitas-saber-para-prevenir-el-cancer-de-prostata

Fundación Femeba. (16 de abril de 2020). El futuro del rastreo de cáncer de próstata con antígeno prostático específico. Recuperado el 25 de 07 de 2020, de https://www.fundacionfemeba. org.ar/blog/farmacologia-7/post/el-futuro-del-rastreo-de-cancer-de-prostata-con-antigeno-prostatico-especifico-47746

Fundación Femeba. (2020). El futuro del rastreo de cáncer de próstata con antígeno prostático específico. Recuperado el 25 de 07 de 2020, de https://www.fundacionfemeba.org.ar/blog/farmacologia-7/post/el-futuro-del-rastreo-de-cancer-de-prostata-con-antigeno-prostatico-especifico-47746\#blog_content
NCl. (4 de Octubre de 2017). Análisis del antígeno prostático específico (PSA). Recuperado el 25 de 07 de 2020, de https://www.cancer.gov/espanol/ tipos/prostata/hoja-informativa-psa

Ormachea, P., Sánchez, R., Callisaya, J., \& Salcedo, L. (Diciembre de 2011). Utilidad del PSA (Antígeno Prostático Específico) total como método de tamizaje para diagnóstico de hipertrofia de próstata y cáncer prostático, Hospital Obrero No 1, febrero-mayo del 2009, Bolivia. BIOFARBO, 19(2), 3944. Recuperado el 25 de 07 de 2020, de http:// www.scielo.org.bo/pdf/rbfb/v19n2/a06.pdf

Panach, J., Gironés, A., Sánchez, E., Doménech, C., \& Martínez, J. (Abril de 2017). Uso del antígeno prostático específico en atención primaria (PSA). Semergen, 43(3), 189-195. doi:10.1016/j. semerg.2016.04.023

Uribe, J. (Diciembre de 2007). Cáncer de Próstata. ¿Qué es el antígeno prostático específico? (La biología del PSA). Urología Colombiana, 16(3), 37-46. Recuperado el 25 de 07 de 2020, de http://www. redalyc.org/articulo.oa?id $=149120470006$

Vachani, C. (18 de Mayo de 2020). Antígeno Prostático Específico (PSA). Recuperado el 25 de 07 de 2020, de https://es.oncolink.org/tipos-de-cancer/ cancer-de-la-prostata/screening-diagnosis/antigeno-prostatico-especifico-psa

Viveros, J., Rojas, L., Solares, M., Lamm, L., Morales, J., C., P., \& F., C. (Agosto de 2015). Utilidad de la fracción libre de antígeno prostático específico como factor pronóstico del estadio patológico en pacientes de prostatectomía radical. Bioreview, 48. Recuperado el 25 de 07 de 2020, de http://revistabioreview.com/revista-nota.php?nota $=193 \&$ revista $=48$

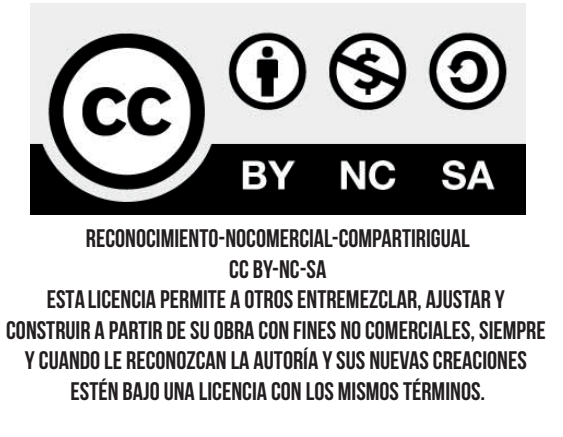

\section{CITAR ESTE ARTICULO:}

Corella Sangui, P., Martínez Otálora, J., Hernández Acosta, Y., \& Cerón Pérez, D. (2020). Utilidad del antígeno prostático específico cáncer de próstata. RECIAMUC, 4(3), 80-89. doi:10.26820/reciamuc/4.(3).julio.2020.80-89 